\title{
Dramas y dilemas en las intervenciones sobre la infancia "en riesgo" en un dispositivo estatal de protección de derechos
}

\section{Natalia Larrea ${ }^{1}$}

1 Universidad Nacional del Centro de la Provincia de Buenos Aires, Olavarría, Argentina Consejo Nacional de Investigaciones Científicas y Técnicas

(iD) https://orcid.org/oooo-0003-1416-392X

Correo electrónico: natalia.larrea@soc.unicen.edu.ar
Recibido:

septiembre de 2020

Aceptado:

enero de 2021

doi: $10.34096 /$ cas.i53.8641

\section{Resumen}

Este trabajo se enmarca en una investigación etnográfica que busca avanzar en la comprensión de las modalidades que asume en la actualidad la protección de la infancia considerada "en riesgo". A partir de un trabajo de campo desarrollado en un dispositivo estatal de protección de derechos, analizo las modalidades de intervención sobre la infancia y sus familias en una localidad de rango medio de la provincia de Buenos Aires. El trabajo se centra en los escenarios dilemáticos que configura la traducción del lenguaje de derechos en intervenciones concretas efectuadas por agentes de este dispositivo. Con ello busco mostrar que los sentidos dados a la protección de la infancia, asociados principalmente a nociones como el consenso, la voluntariedad y la responsabilidad familiar, se tensionan en la práctica concreta y situada resignificando el lenguaje de derechos en la interacción entre familias y Estado.

\section{Dramas and dilemmas in interventions on children "at risk" in a state agency for rights protection}

\begin{abstract}
This article is part of ethnographic research that seeks to contribute to the understanding of the modalities of protection of children considered "at risk". Based on fieldwork conducted in a state agency for the protection and restitution of children's rights, it analyzes the modalities of intervention on this population and their families, in a midsized locality of the province of Buenos Aires. The paper focuses on the dilemmatic scenarios that shape the translation of the language of rights into concrete interventions carried out by state agents. It is argued that the meanings assigned to child protection,
\end{abstract}

\section{Palabras clave}

Derechos de niñas, niños y adolescentes; Dispositivo estatal; Protección de derechos; Estado; Familias

\section{Key words}

Children's rights; State agency; Protection of rights; State; Families 


\begin{abstract}
.
\end{abstract}
mainly associated to notions such as consensus, voluntariness and family responsibility, are put under strain in concrete and situated practice by resignifying the language of rights in the interaction between families and the State.

\title{
Dramas e dilemas em intervenções sobre crianças "em risco" em um órgão estadual de proteção de direitos
}

\section{Resumo}

Palavras-chave

Direitos da criança; Agência estadual; Proteção dos direitos; Estado; Famílias
1. En adelante se mencionará a este tratado por sus siglas, CIDN, o simplemente como Convención.
2. La noción de tutela estatal cobra centralidad en el contexto de la Ley de Patronato de Menores, sancionada en la Argentina en el año 1919. Villalta (2012) señala que las explicaciones más comunes sobre el surgimiento del Patronato de Menores como entramado jurídico-administrativo de tutela estatal sobre la población infantil hacen foco en el contexto de urbanización creciente e inmigración masiva de las primeras décadas del siglo XX, y enfatiza que "ella surge como una respuesta del Estado a la necesidad ya sea de 'proteger' a un número cada vez mayor de

niños pobres y abandonados o bien de 'extender el control' sobre las capas más pobres de la población"

(p. 79).

3. Refiero a la Ley Nacional $\mathrm{N}^{\circ}$ 26.061 y Ley Provincial $N^{\circ} 13.298$.
Este trabalho é parte de uma pesquisa etnográfica que procura avançar no entendimento das modalidades atualmente assumidas pela proteção das crianças consideradas "em risco". Com base no trabalho de campo implementado em uma agência estadual para a proteção e a restituição dos direitos da criança, são analisadas as modalidades de intervenção sobre esta população e suas famílias em uma localidade de médio alcance da província de Buenos Aires, Argentina. O trabalho se concentra nos cenários dúbios que moldam a tradução da linguagem dos direitos em intervenções concretas realizadas pelos agentes desta agência. Se argumenta que os significados atribuídos à proteção da criança, principalmente associados a diferentes noções como consenso, voluntariedade e responsabilidade familiar, são enfatizados na prática concreta e situados através da ressignificação da linguagem dos direitos na interação entre as famílias e o Estado.

\section{Introducción}

Este trabajo propone una reflexión que toma como contexto las transformaciones legislativas e institucionales que, al calor de la institucionalización de los postulados de la Convención Internacional de los derechos del Niño (CIDN) ${ }^{1}$ en la Argentina, y al enfoque de derechos en ella cristalizado, cimentaron y dieron forma a las actuales modalidades de administración de problemáticas asociadas a la infancia considerada "en riesgo". En particular, busco indagar en los modos en que la adecuación del enfoque de derechos en contextos donde la desigualdad social constituye la particularidad sobresaliente, configura escenarios dilemáticos para quienes deben intervenir sobre la infancia y sus familias que tensionan los sentidos dados a la protección.

Las adecuaciones locales del enfoque de derechos implicaron complejas transformaciones normativas e institucionales que instalaron gradualmente a nivel global una retórica sobre la niñez y sus derechos. En la Argentina, esta retórica fue construida en clara contraposición a la mirada que se había instalado sobre la niñez en el contexto del "sistema tutelar", erigido en torno a la cristalización de la categoría de "minoridad" (Villalta, 2012). A su vez, ciertos tópicos presentes en la CIDN se ponderaron a la par que otros fueron invisibilizados (Barna, 2014a). Así, la figura de niñas y niños sujetos de derechos y ya no objetos de tutela estatal, ${ }^{2}$ la desjudicialización de problemáticas asociadas a contextos de pobreza, la desinstitucionalización de niñas, niños y adolescentes y la entronización de la familia, fueron las directrices salientes de esta retórica en el país. En este sentido, el rasgo distintivo que conlleva la adecuación normativa a la CIDN, y específicamente a las leyes de protección integral de derechos en la Argentina, ${ }^{3}$ radica en el traspaso de históricas competencias y facultades propias del Poder Judicial sobre la infancia y sus familias, al ámbito de la administración pública. Sin embargo, varios estudios (Villalta, 2010; Grinberg, 2013; Barna, 2014a; Villalta y 
Llobet, 2015) han advertido sobre los riesgos de asumir una representación dicotómica entre lo que ha sido denominado el paradigma de la "situación irregular" en contraposición al de la "protección integral de derechos" en tanto puede conllevar una esencialización e invisibilización de continuidades entre uno y otro. ${ }^{4}$

Ahora bien, el lenguaje de los derechos concebido como lenguaje moral (Schuch, 2008; Vianna, 2010) que orienta las prácticas de quienes deben adecuarlo a sus intervenciones cotidianas, se ve muchas veces tensionado en su traducción situacional. Y ello porque, tal como señalan Fonseca y Cardarello (2005), los derechos en su forma abstracta y descontextualizada poco significan. En tal sentido, analizar las tensiones surgidas de las prácticas reales que encarnan los derechos puede ofrecer valiosas pistas para la comprensión de las dinámicas locales de intervención estatal sobre la infancia y sus familias. Asimismo, como señala Merry (2010), existe una tensión inevitable entre los principios generales de los derechos humanos y las situaciones particulares en las cuales deben implementarse:

El derecho de los derechos humanos, como sistema legal, se propone aplicar uniformemente principios universales a todas las situaciones. No piensa sus intervenciones en función de situaciones sociales y políticas concretas, aun cuando esas situaciones podrían inspirar diferentes enfoques sobre la justicia social [...] Esta distancia entre las visiones globales de la justicia y las visiones específicas de los contextos locales crea un dilema fundamental para la práctica de los derechos humanos. (p. 86)

A partir de estas consideraciones, este trabajo propone mostrar que los sentidos que envuelven las prácticas concretas de protección de la infancia, asociados al lenguaje moral de los derechos, se tensionan en el marco de las intervenciones concretas, resignificándolo. Sentidos asociados, fundamentalmente, a las ideas de voluntariedad, consenso y responsabilidad de las familias, aparecen imbricados en modalidades que incluyen técnicas de negociación y construcción de acuerdos entre profesionales y familias destinatarias de las intervenciones, como modo de resolución de las problemáticas. Sin embargo, no por ello dejan de representar lo que podría caracterizarse como formas estilizadas del ejercicio de poder propio de la dimensión tutelar del Estado (Pacheco de Oliveira, 1988; Souza Lima, 2009). Así, cuando me refiero a la complejidad de ciertas situaciones y problemáticas intento hacer alusión, por un lado, a una configuración de intervenciones en la que el lenguaje moral de los derechos, enaltecido por sobre ciertos elementos asociados a relaciones de carácter tutelar con los cuales convive, se inscribe en contextos signados por la desigualdad social y la precariedad institucional. ${ }^{5}$

A partir de la reconstrucción de un caso caracterizado como complejo por quienes estaban a su cargo en un organismo de protección de derechos, analizo los diferentes escenarios dilemáticos configurados mediante las intervenciones efectuadas. Esta situación se revelaba fructífera para mi indagación de campo en tanto resultaba paradigmática, no solo por la relevancia que adquirió en el ámbito del organismo a raíz de un suceso trágico que involucraba a un adolescente, sino porque el suceso había desencadenado una serie de prácticas e intervenciones que tensionaban el repertorio moral del discurso de los derechos, develaban limitaciones y contradicciones en su adecuación institucional.

Si bien los dilemas que las situaciones exponen no resultan exclusivos de este caso en concreto, observarlos desde la particularidad de las intervenciones aquí implementadas permite iluminar prácticas que, inscriptas en mecanismos de intervención contemporáneos, conjugan al mismo tiempo elementos de más larga data. Se trata de escenarios de actuación donde las estrategias de protección implementadas no resultan eficaces y
4. Por otra parte, Villalta (2013) señala que, si bien esta concepción antagónica sirvió como herramienta de denuncia de violaciones a los derechos humanos de niñas, niños y adolescentes en la Argentina, concebirla de esta manera imposibilita comprender las condiciones y relaciones sociales que daban lugar a intervenciones abusivas y discrecionales.
5. Refiero a una precariedad en términos de recursos materiales $y$ humanos que pude constatar por medio del relato de los propios profesionales del organismo donde desarrollé el trabajo de campo, pero principalmente a partir de la cotidianeidad de la que fui parte. 
6. La localidad se encuentra ubicada en el centro-oeste de la provincia de Buenos Aires, encuadrándose y se encuadra como una ciudad de rango medio, siendo con una población de aproximadamente 119.441 habitantes (INDEC, 2010).

7. Resulta importante aclarar que, con vistas a una mejor y más acabada reconstrucción del caso y atendiendo a los fines analíticos propuestos, recurrí a la posibilidad de actualizar información sobre

el mismo al iniciar una nueva

instancia de trabajo de campo enmarcada en una investigación doctoral a fines de 2019.

8. El genérico masculino refiere a su denominación original en la letra de la ley.

9. El uso de comillas se utilizará para dar cuenta de categorías y nociones propias del campo, así como para identificar la voz de los actores y referencias documen- debe dirimirse el destino de un adolescente cuando su ámbito familiar es considerado "peligroso". Este trabajo, entonces, procura dar cuenta de los elementos que intervienen y se conjugan al momento de adecuar las intervenciones a estos escenarios.

Desarrollé mi trabajo de campo en el ámbito del Servicio Local de Promoción y Protección de Derechos de Niños, Niñas y Adolescentes de una localidad del centro de la provincia de Buenos Aires, ${ }^{6}$ por un período de cinco meses entre abril y septiembre de 2018. Ello implicó mi presencia y participación en las distintas prácticas y actuaciones desplegadas por agentes de este dispositivo estatal: entrevistas, reuniones de equipo, visitas a hogares familiares, reuniones con actores institucionales. Asimismo, tuve la posibilidad de acceder a la documentación que se produce cotidianamente allí y también pude sostener diversas entrevistas informales con trabajadores del organismo. Los legajos de niñas, niños y adolescentes fueron también objeto de indagación y fuente primordial en la reconstrucción del caso que nutre este trabajo. ${ }^{7}$

\section{El trabajo con las familias en un Servicio Local de Promoción y Protección de Derechos de Niños, Niñas y Adolescentes}

La implementación en la provincia de Buenos Aires de la Ley N 13.298 de Promoción y Protección Integral de Derechos de Niñas, Niños y Adolescentes significó una profunda transformación del entramado institucional destinado a la protección de la infancia. El Sistema de Protección Integral de Derechos que comienza a configurarse enmarcado en esta normativa supuso la creación a nivel municipal de los denominados Servicios Locales de Promoción y Protección de Derechos del Niño. ${ }^{8}$ Los equipos técnicos que componen los Servicios Locales — profesionales del campo del trabajo social, la psicología, la abogacía y la sociología, entre los más comunes- tienen por meta proteger y restituir derechos mediante la implementación de programas y planes destinados a esta población y de un abordaje de las problemáticas centrado en lo que allí denominan el "trabajo con las familias"."

Quienes desempeñan su labor en estos dispositivos consideran que por medio de la modificación y/o reversión de conductas y comportamientos considerados "riesgosos" en el entorno familiar de un niño, niña o adolescente, se restituirían sus derechos vulnerados. En razón de ello, el fin último del trabajo desarrollado con las familias es la producción de un "orden familiar" (Ciordia y Villalta, 2012: 436), mediante la "normalización" de comportamientos asociados a los roles maternos y paternos - aunque principalmente maternos - considerados "desviados". Para ello, los formatos de intervención implementados se basan en el diálogo, la persuasión y la negociación más que en la mera imposición (Vianna, 2010; Villalta, 2010; Barna, 2014a).

La labor desarrollada por el equipo técnico del Servicio Local de la localidad de estudio se divide entre lo que puede definirse como prácticas de intervención sobre las familias o "atención de casos" y, por otro lado, la producción de documentos. Esto último constituye una tarea que ocupa un lugar fundamental en la cotidianeidad del organismo, ya que permite hacer legible el accionar y los formatos de intervención que allí se despliegan (Barna, 2014b).

Asimismo, resulta una condición insoslayable para quienes llevan a cabo las intervenciones, lograr el compromiso y la responsabilidad de las familias en el abordaje de las problemáticas, en tanto es considerada parte corresponsable en esta tarea. Si bien la idea de corresponsabilidad abarca un espectro multiactoral que alcanza variados ámbitos estatales y de la sociedad civil, son las familias, principalmente, las interpeladas a participar en la cogestión de la infancia (Barna, 2014c). Es en este sentido que muchas 
veces el ideal de la corresponsabilidad resulta tensionado cuando la propia familia, contraparte fundamental, es considerada un peligro para la niña, niño o adolescente.

\section{El drama de Manuel ${ }^{10}$}

Supe del caso que a continuación reconstruyo durante mi estadía en el Servicio Local, a partir de un suceso acaecido en marzo de 2018 relacionado con Manuel, un adolescente de catorce años con quien desde el organismo intervenían desde mediados de 2015 por problemáticas definidas como "conflictivas vinculares" entre miembros de su familia. Sin embargo, el problema específico que se buscaba revertir, en principio, eran las "conductas riesgosas" del adolescente asociadas a lo que allí definían como "consumo problemático".

Manuel vivía junto a su mamá, dos hermanas de once y catorce años respectivamente, dos tías paternas y una prima de trece años en una precaria vivienda ubicada en un barrio periférico de la localidad. Su padre había sido víctima de un homicidio ocho años antes, razón por la cual su mamá se encontraba en tratamiento psiquiátrico desde entonces. Los únicos ingresos económicos de la familia correspondían a subsidios otorgados por el Estado y a empleos esporádicos informales, principalmente de sus tías. Al momento de las primeras intervenciones del Servicio Local sobre el caso, la vivienda había sido incendiada, aparentemente, por una tía y primos maternos que viven en el mismo barrio, con quienes tanto Manuel como su grupo familiar conviviente mantenían asiduas peleas y conflictos. De este modo, luego del incendio, la familia decide mudarse a la casa de la abuela materna del adolescente, residente del mismo barrio. Allí viven la mujer junto a su pareja, quien realiza trabajos de albañilería, una hija de ocho años y una nieta de nueve que se encontraba bajo su cuidado luego del suicidio de su madre, dos meses antes. La hermana de esta niña es quien se encontraba al cuidado de la mamá de Manuel y vivía junto a él y su familia en la vivienda incendiada. Si bien el municipio les otorgó materiales para la reconstrucción de la casa, la mamá de Manuel decía no conseguir mano de obra accesible que les permitiera avanzar con la tarea. Por lo que, durante el tiempo que abarca la reconstrucción del caso que aquí propongo, el grupo familiar se encontraba aún conviviendo en la casa de la abuela materna de Manuel.

Reconstruyo el caso a partir de conversaciones informales con quienes eran sus responsables en el Servicio Local, la indagación documental -específicamente del legajo de Manuel- ${ }^{11}$ y la observación de una instancia de tratamiento terapéutico. A los fines analíticos de este trabajo se dividirá el caso en tres momentos o instancias de desarrollo. El primero de ellos se ubica en las primeras intervenciones - mediados de 2015- hasta el evento dramático de un homicidio, en abril de 2018, e incluye el recorrido por los escenarios de implementación de estrategias que buscaban involucrar y comprometer a la familia del adolescente. El segundo momento se extiende desde la institucionalización temporal de Manuel en abril de 2018, hasta el momento en que se definen las condiciones para su eventual egreso en el mes de junio. Aquí focalizaré en el modo en que los agentes involucrados dirimen estas condiciones y producen responsables por la situación del adolescente. Por último, el tercer momento se desarrolla a partir de su egreso, a fines de 2018, hasta su ingreso a una comunidad terapéutica hacia finales de 2019. Aquí el foco estará puesto en los usos dados a la institucionalización tanto por agentes como por su familia.

\section{La búsqueda de voluntariedad y consenso}

A mediados del año 2015 el Servicio Local recepciona un oficio judicial desde el Juzgado de Familia de la localidad, por medio del cual se solicita su intervención por motivo de estar involucrado Manuel. Una denuncia efectuada por su madre, a raíz
10. Tanto el nombre del adolescente implicado en el caso que se reconstruye como los de familiares y actores del Servicio Local mencionados en este trabajo son ficticios, apelando al resguardo de su identidad.

11. El escrutinio documental constituye un método de indagación etnográfica que en el marco de este ámbito institucional tiene mucho que aportar. Por un lado, a los fines de recrear el caso en sus distintas temporalidades, más allá de lo que los actores institucionales puedan narrar sobre él. Por otro lado, debido a que pueden ser concebidos como un campo de indagación en sí mismos, situándolos en las lógicas institucionales que les dieron origen y explorando así los sentidos con los que fueron dotados (Muzzopappa y Villalta, 2011). 
del incendio intencional de su vivienda por parte de un familiar cercano, había sido el origen de apertura de una causa judicial. Días más tarde llega al Servicio una "solicitud de intervención" por parte de la institución educativa a la que asistía Manuel, que informaba principalmente "una situación de cambio de conducta, a nivel escolar, que ha experimentado el niño durante este último tiempo, lo cual afecta sustancialmente su rendimiento escolar, y una amplia vulneración de derechos como ser a la salud y la vivienda" (Fragmento de "informe de Dirección de Psicología Comunitaria y Pedagogía Social y Dirección de Educación Primaria”, noviembre de 2015, extraído del legajo del adolescente, p. 4). Durante las primeras intervenciones - entrevistas con la mamá, otros familiares y actores de la institución educativa-, los profesionales responsables del caso habían identificado una "imposibilidad de su familia para poder acompañarlo y contenerlo". Asimismo, expresaban: "Manuel siempre se mostró reticente a cualquier tipo de asistencia de nuestra parte"; "nunca pudo implicarse en su situación problemática y comprometerse a partir de las indicaciones y herramientas que le dimos"; "esta actitud responde a una problemática que no es de él solo, que es de su grupo familiar". En tal sentido se identificaban "escasos recursos", asociado esto a que sus miembros - fundamentalmente su madre y dos de sus tías- "no lograban sostener las indicaciones sugeridas para que pudieran modificar sus modos de vinculación violenta y conflictiva". Estas indicaciones tenían que ver, sobre todo, con lograr que tanto el adolescente como su madre asistieran a tratamientos terapéuticos y, fundamentalmente, que Manuel concurriera al Centro de Prevención de Adicciones (CPA) sugerido desde el Servicio Local.

Durante el transcurso de dos años la "situación problemática" no se revertía; ni Manuel ni su madre asistían a las citaciones en el organismo, en las visitas domiciliarias casi nunca se los encontraba y no lograban regularidad en los tratamientos terapéuticos. En el Servicio asociaban esto a lo que entendían como una "escasa voluntad" para revertir el problema. La madre de Manuel expresa en una de las entrevistas realizadas que "no puede convencer a su hijo, que se le escapa de las manos", pero para quienes llevaban a cabo el abordaje del caso - Laura y Felipe, trabajadora social y psicólogo del Servicio- la escasa voluntad se presentaba tanto en Manuel como en toda su familia, y se aludía a su madre y tías paternas, quienes mantenían una relación muy estrecha. Así lo expresa uno de los informes elaborados sobre el caso: "Se evalúa que el joven presenta resistencias vinculadas mayoritariamente con la problemática de su grupo familiar de acompañarlo y contenerlo emocionalmente" (Fragmento de "informe de situación", marzo de 2018, extraído del legajo del adolescente, p. 21).

La situación de Manuel se complejizaba no solo porque no conseguían regularidad en su asistencia al tratamiento terapéutico sugerido, principal estrategia implementada, sino porque su madre manifestaba recurrentemente "no poder con su hijo". Y aquí se presenta uno de los dilemas centrales a la hora de efectuar las intervenciones, puesto que las estrategias que se despliegan aspiran a la voluntariedad de las familias como

12. Apelando a la dimensión moral del enfoque de derechos, el autor plantea que ciertos imperativos que de allí emanan se axiomatizan creando efectos de sentido que inciden en las actuaciones estatales de intervención sobre niñas, niños y adolescentes.

13. La jornada laboral habitual en el Servicio Local se extiende desde las 7 hasta las 13, de lunes a viernes. Simultáneamente funciona

la guardia de emergencia todos

los días, durante las 24 horas, por medio de una línea telefónica exclusiva, cuya responsabilidad de atención se establece por turnos rotativos del equipo técnico. horizonte moral (Barna, 2014c) ${ }^{12}$ donde priman actuaciones consensuadas y se limita el carácter compulsivo que pudieran adquirir, a situaciones de extrema gravedad. De modo que los esfuerzos de Laura y Felipe se dirigían principalmente a tratar de convencer a Manuel de que lo mejor para él era que asistiera al espacio terapéutico sugerido. Pero para ello también resultaba necesario contar con los esfuerzos de su madre, esto es, lograr que se comprometiera a apuntalar tanto las conductas de su hijo como las propias.

Durante los primeros días de marzo de 2018 el Servicio Local recibe un llamado en horas de guardia, ${ }^{13}$ proveniente del área de pediatría del Hospital Municipal. Desde allí se solicita intervención debido a la internación de Manuel por una herida de gravedad que le había provocado su tío materno, a raíz de una pelea. Laura y Felipe se acercan al hospital y allí se notician de que el adolescente atravesaba un cuadro de 
"excitación psicomotriz producida por el consumo de clonazepam y alcohol" (Fragmento de "informe de situación", marzo de 2018, extraído del legajo del adolescente, p. 21), previo a la pelea. Allí mismo entablan un diálogo con la mamá de Manuel y su tía paterna, quienes acompañaban al adolescente, y acuerdan una visita a su hogar para poder "establecer acuerdos sobre el tratamiento que desde el Servicio Local se sugeriría" (Fragmento de "informe psicológico" del Servicio de Pediatría, área salud mental, hospital municipal, marzo de 2018, extraído del legajo del adolescente, p. 30). Es durante esta visita, concretada una vez que Manuel regresa a su hogar, que Laura y Felipe logran convencerlo de ingresar nuevamente al espacio del CPA y le sugieren, además, el ingreso a un centro de día. ${ }^{14}$

Sin embargo, el desenvolvimiento de las estrategias propuestas continúa sin "éxito" debido a que "no resulta posible lograr que la madre de Manuel pueda implicarse y generar contención y acompañamiento a su hijo" (Fragmento de "acta de entrevista" a la madre de Manuel en sede del Servicio Local, marzo de 2018, extraído del legajo del adolescente, p. 24). De todas maneras, Laura y Felipe insisten por el ingreso de Manuel al CPA y deciden acompañarlo ellos mismos durante los primeros encuentros en este marco institucional. Si bien hasta aquí las intervenciones tendían a reforzar los acuerdos establecidos y consensuar estrategias de intervención, su efectivización recaía meramente en la voluntad de Manuel y su familia. Sin embargo, el ingreso del adolescente al hospital pareciera haber redefinido la relación de aparente flexibilidad que durante este tiempo había permeado el vínculo agentes/familia para pasar a asumir, los primeros, la responsabilidad por la asistencia de Manuel al espacio terapéutico sugerido.

A inicios del mes de abril, nuevamente el caso "se cae", como acostumbran referir en el Servicio Local a las medidas e intervenciones que no resultan eficaces. Desde el centro de día se comunican para informar que el adolescente no asistía a la institución y que además había "manifestado conductas transgresoras" (Diario de campo, agente del Servicio local, julio de 2018), refiriendo específicamente a amenazas dirigidas a miembros del equipo institucional. Durante el transcurso de esa misma semana, el Servicio Local es notificado, mediante un oficio judicial del Juzgado de Garantías del Joven, ${ }^{15}$ de un homicidio protagonizado por Manuel, quien había disparado con un arma de fuego a su tío. Este hecho causó gran conmoción en el organismo debido a que, si bien el caso no se dirigía hacia los objetivos previstos y el adolescente no lograba ser apuntalado, no se esperaba semejante suceso. A raíz de este episodio, el Juez de Garantías del Joven dictamina una medida de seguridad, ${ }^{16}$ con alojamiento temporal en un centro de contención de régimen cerrado, hasta tanto considere garantizadas las condiciones para revertir tal situación.

Más allá de que desde un primer momento tanto Manuel como su familia reconocieran la necesidad de revertir una situación caracterizada como problemática, asociada a sus relaciones familiares, no se logró sostener un compromiso para modificarla. El énfasis en el tratamiento estaba puesto en la preservación de los valores de la armonía y la responsabilización individual en la conducción de las respuestas al problema (Schuch, 2008). Como propone Grinberg (2018), quienes llevan adelante el denominado trabajo con las familias desarrollado en estos organismos no pretenden imponer por la fuerza, sino que buscan transformar situaciones y personas a partir de acciones que apelan a la empatía, la proximidad y el consenso, involucrando a los sujetos como responsables de su propia transformación. En tal sentido, las estrategias de intervención implementadas en el caso de Manuel buscaron, sobre todo, construir y reforzar una responsabilización ante la problemática, tanto en el adolescente como en su madre.
14. Espacios institucionales dependientes de los gobiernos municipales y también de organizaciones de la sociedad civil, que brindan atención integral a niñas, niños y adolescentes. Allí se desarrollan actividades, durante gran parte del día y bajo la modalidad de talleres, de capacitación en oficios, recreativas, de índole terapéutica $y$ asistencia en tareas escolares, entre otras. En la localidad en la que se enmarca este trabajo, quienes asisten a estos espacios generalmente lo hacen por sugerencia o derivación de instituciones educativas, de salud y del Servicio Local, pero también pueden hacerlo por demanda espontánea e intermediación de referentes barriales.

15. El marco normativo que crea y regula el Fuero de Responsabilidad Penal Juvenil en el ámbito de la provincia de Buenos Aires - Ley 13.634- establece la constitución de los Juzgados de Garantías del Joven. Estos organismos se ocupan del control de la legalidad de los procesos tramitados ante este fuero y son responsables de las medidas que se tomen con respecto a jóvenes acusados de cometer delitos.

16. Medida restrictiva de libertad ambulatoria, provista por la Ley 13. 634, para los casos que involucran menores de 16 años, considerados no punibles por el régimen de administración judicial para jóvenes en Argentina. Esta medida puede dictaminarse considerando la gravedad del delito, por disposición del juez de Garantías del Joven. 


\section{La construcción de responsables}

17. En tanto la medida de seguridad es implementada de manera provisoria, el juez de Garantías del Joven deberá arbitrar los medios necesarios para establecer la condicionalidad de su supresión. De este modo, solicita al Servicio Local que disponga de medidas de su competencia, considerando las previas intervenciones del organismo sobre el caso de Manuel, en los términos que el artículo 63 de la Ley $N^{\circ} 13.634$ dispone: "el Juez de Garantías establecerá la pertinencia de aplicar alguna de las medidas de Protección Integral de Derechos establecidas en la Ley

$N^{\circ} 13.298$, en cuyo caso solicitará la intervención del Servicio de Protección de Derechos correspondiente".

18. Los informes de situación constituyen documentos de elaboración constante en el Servicio Local. Allí se plasma la biografía de un caso, incluyendo intervenciones efectuadas y caracterización de las personas involucradas. Estos informes, además de constituir

legajo del caso, circulan por medio de las articulaciones interinstitucionales enmarcadas en el principio de corresponsabilidad que establece la normativa - Ley

No $13.298-y$ pueden ser elaborados a demanda de otras instituciones, o a la inversa, cuando es el Servicio Local quien solicita la intervención de otra institución sobre el caso.
Por medio del oficio judicial que notifica sobre el homicidio, el juez de Garantías del Joven cita a personal del Servicio Local a asistir a una audiencia con el fin de disponer estrategias alternativas a la actual medida de seguridad adoptada. ${ }^{17} \mathrm{~A}$ esta audiencia asisten el director del Servicio Local y Felipe, Manuel, su madre, dos tías y su abuela paterna. La sugerencia realizada al juez por el director y Felipe, para suplantar la medida de seguridad adoptada, no distaba de lo ya implementado anteriormente. El director expresa que podrían ofrecerle "un programa de centro de día con traslado institucional, un espacio de contención - CPA - y de tratamiento psicológico." (Fragmento de oficio judicial: acta de audiencia en Juzgado de Garantías del Joven № 1 de Azul, mayo de 2018, extraído del legajo del adolescente, p. 59). A su vez, la abuela paterna de Manuel expresa que está dispuesta a que el adolescente conviva con ella por el tiempo que el juez disponga, a fin de que no regrese a su hogar, para evitar así "posibles represalias por parte de miembros de la familia materna" (Diario de campo, agente del Servicio Local, julio de 2018) allegados al tío del adolescente. Felipe expresa que desde el instituto en el cual se encontraba alojado Manuel manifiestan gran preocupación por un eventual egreso, debido a que ven en la familia una potencial "amenaza a los avances del adolescente" (Diario de campo, agente del Servicio Local, julio de 2018). Estos avances se asociaban, fundamentalmente, a que Manuel había logrado "problematizar su situación e involucrarse en un tratamiento psicológico" (Fragmento de "acta telefónica" entre agente del Servicio Local y responsable del centro de contención, junio de 2018, extraído del legajo del adolescente, p. 58).

A mediados de junio se realiza una nueva audiencia judicial a fin de resolver el posible egreso del joven. Desde el instituto se envían algunos informes "positivos" con respecto a Manuel, pero se expresa preocupación sobre la posibilidad del retorno a su hogar. Finalmente, en el marco de la audiencia en la cual se decidiría su destino, el juez determina la continuidad de la medida de seguridad que se impusiera inicialmente. En un informe de situación ${ }^{18}$ sobre el caso, elaborado desde el Servicio local, se expresa:

El juez que intervino en la causa entendió que, a pesar de los informes positivos del equipo profesional de la institución donde se encuentra alojado el joven, el grupo familiar que lo alojaría, de producirse su vuelta a la localidad, aún no ha demostrado un avance respecto del trabajo en relación a la resolución conflictiva y violenta de los conflictos interpersonales, según lo informado por el Servicio Local. (Fragmento de “informe de situación”, junio de 2018, extraído del legajo del adolescente, p. 64).

De esta manera solicita al Servicio que "disponga de las medidas necesarias a fin de lograr un egreso favorable para Manuel" (Fragmento de "acta general de audiencia”, junio de 2018, p. 64). Resulta importante en este punto señalar el modo en que todas estas disposiciones moldean percepciones asociadas al fracaso y a una culpabilización que los profesionales viven bajo la forma de dramas personales (Schuch, 2008), lo que pone en evidencia el modo en que estos agentes se enfrentan a las situaciones que gestionan sin las condiciones necesarias para garantizar la protección de derechos. Como señala Barna (2014b), esta contradicción impone a los agentes la necesidad de generar mecanismos precarios e informales de clasificación de personas y estimación de atributos familiares para lograr estructurar las intervenciones e intelegibilizar un escenario de actuación. Así es como desde el Servicio Local consideran que la familia de Manuel - su madre, sus dos tías paternas y su abuela materna- deberían participar de un espacio de tratamiento terapéutico denominado Terapia Multifamiliar, "a fin de poder adquirir recursos para resolver sus problemáticas de manera armónica” (Fragmento de “informe de situación”, junio de 2018, extraído del legajo del adolescente, p. 65). 
El espacio de Terapia Multifamiliar constituye una modalidad terapéutica de abordaje de diversas problemáticas asociadas principalmente a lo que en el Servicio denominan "conflictivas vinculares". Dicho espacio es ofrecido por el área de salud mental del hospital municipal y está destinado a personas derivadas allí "para resolver sus modos de vinculación violenta" (Diario de campo, Servicio Local, julio de 2018), como suele mencionarse en el Servicio a la tarea llevada adelante en este espacio.

El primer día de asistencia de la madre y tías de Manuel al espacio que tuve la posibilidad de observar (su abuela no concurrió), la profesional a cargo les pide que se presenten ante los demás participantes y relaten el motivo por el cual se encontraban allí. La mamá del adolescente comienza su relato en voz baja con su mirada agachada inmediatamente comienza a llorar, por lo que una de las tías continúa el relato narrando los motivos de su asistencia al espacio. Menciona que su sobrino "había hecho algo muy malo" y define este episodio como una "venganza". Seguidamente, narra el modo en que sucedió el homicidio enfatizando que "Manuel ya estaba cansado" y que "ya venía arrastrando cosas". Entre lágrimas, tanto la tía como la mamá expresan que quieren que Manuel "se reintegre", que "le den una oportunidad, porque es muy chiquito". (Diario de campo, espacio de Terapia Multifamiliar, julio de 2018).

La medida de seguridad es implementada, según Laura y Felipe, como forma de evitar que Manuel vuelva a cometer otro hecho delictivo y al mismo tiempo que la familia del tío "tome represalias contra él". De esta manera, además de responsable, la familia del adolescente representaba un potencial "peligro" y, en razón de esto, la medida de seguridad tomaba la forma de una aparente medida de protección. En tal sentido, las intervenciones de aquí en más se dirigirían a erradicar el peligro representado en la familia de Manuel.

Douglas (1996) señala que nuestras representaciones de "peligro" y "riesgo" se basan en ideas de moral y justicia que caracterizan ciertos elementos como peligrosos al tiempo que niegan, olvidan u ocultan otros. Para la autora, existe una selección cultural de peligros que implica la percepción de ciertos riesgos para su eventual prevención, mientras que otros son descuidados y pasan desapercibidos. De este modo, ciertos principios morales legitiman la percepción de riesgos guiando la respuesta social dada. En este sentido, si el ámbito familiar de Manuel era considerado peligroso, este peligro estaba asociado a la idea de violencia que permeaba las relaciones familiares y entonces, los riesgos que se advertían y se buscaban prevenir tenían que ver con la permanencia del adolescente en su ámbito familiar. Grinberg (2008) sostiene:

\section{A diferencia de la noción de peligro, la idea de riesgo, aplicada a la infancia, implicaría un juicio a futuro, un pronóstico realizado en base a la observación de ciertos factores a partir de los cuales se estima que si no se toman medidas necesarias, 'algo malo' le ocurrirá al niño. (p. 159, énfasis en el original)}

Es así que tanto la idea de riesgo como la de peligro, asociadas a la familia de Manuel, configuraban un modo de accionar frente a la situación abordada que orientaba las decisiones hacia medidas que implicaban la separación del adolescente de su ámbito familiar. Evitar el contacto de Manuel con su familia, separándolo de ella e instándola a transitar una instancia terapéutica de autotransformación, se presentaba como la opción adecuada a los efectos de erradicar el peligro. De este modo, el tratamiento terapéutico propuesto al grupo familiar con el fin de corregir sus conductas y comportamientos toma la forma de condición para lograr la desinstitucionalización de Manuel.

Ahora bien, la construcción de responsables por la situación del adolescente, objetivada en su familia, parecía cargar sobre ella una especie de culpabilidad y condena 
por el peligro que representaba para Manuel, pero al mismo tiempo dicha familia era ponderada como lugar al que el adolescente debía regresar. Lo que pareciera resultar una contradicción en el modo en que se efectúan las intervenciones resulta, sin embargo, inherente a los modos actuales de gestión institucional de la niñez. Si en un primer momento la principal situación que se buscaba revertir eran las "conductas transgresoras" y la "problemática de consumo" del adolescente, al momento de la institucionalización estas se tornan subsidiarias de una dificultad mayor que las contiene: un entorno familiar "conflictivo". Así, la familia pasaba a ser la principal responsable por la situación de Manuel y objeto primordial de regulación. De este modo, al ubicar el problema en las relaciones y conductas familiares "inadecuadas" y "riesgosas", es la propia familia la que debe asumir la responsabilidad por la medida adoptada con el adolescente.

\section{Los usos de la institucionalización: entre recursos de protección y estrategias de las familias}

Durante una entrevista en el Servicio Local, posterior a la implementación de la medida de seguridad de Manuel, su madre relata que una trabajadora social del juzgado (sin poder precisar mayores datos) visitó la casa de la abuela paterna del adolescente. Durante la audiencia en la cual el juez decide continuar la medida de seguridad la mujer había ofrecido su hogar para alojarlo. El examen de la vivienda se realizaba con el objetivo de evaluar la posibilidad de que el adolescente residiera en ese domicilio una vez egresase de la institución. La madre manifiesta estar de acuerdo con esta sugerencia ya que la proximidad de residencia con respecto a familiares y allegados al tío del adolescente le hace temer posibles represalias contra Manuel o su familia. De este modo asiente y propone ella misma que se aloje en casa de su abuela, y aclara que, aunque tiene intenciones de "recibir a su hijo", considera que no puede resguardarlo (Diario de campo, entrevista a la madre de Manuel en sede del Servicio Local, 31 de mayo de 2018).

Asimismo, durante una entrevista posterior a la referenciada anteriormente, luego de las primeras sesiones en el espacio de Terapia Multifamiliar, manifiesta que "necesita adquirir más herramientas para poder controlar a su hijo" principalmente porque "ha entendido que, para que pueda regresar a su domicilio, su grupo familiar no debe presentar conflictos" (Fragmentos de "informe de Terapia Multifamiliar", junio de 2018, extraído de legajo del adolescente, p. 67). Hasta aquí resulta evidente que la madre de Manuel ha asumido - o al menos así pretende demostrarlo- el lugar de responsable que le ha sido atribuido por la situación de Manuel. Sin embargo, la opción de la vivienda de la abuela paterna se presentaba como un recurso que podía suplantar la condición impuesta para su egreso, esto es, "transformar sus modos de vinculación conflictivos". Ello porque parecía representar una mejor opción ante la alternativa de que Manuel continuara institucionalizado.

Durante el mes de agosto, el Servicio Local se notifica de que la medida de seguridad sería cesada ante el pedido de revocación de la abogada defensora en la Cámara de Apelaciones. De este modo se dispone que Manuel se aloje en casa de su abuela paterna, tal como habían acordado con su mamá. Además de ello se acuerda también que concurra a un Centro de Día y se reintegre a un espacio escolar, lo cual fue registrado

19. Las actas-acuerdo no solo se efectúan entre agentes y familias, sino también entre instituciones que se abocan conjuntamente al abordaje de un caso, y en el Servicio Local se las denomina "actas de corresponsabilidad”. Así, en estos documentos se plasman por escrito las actuaciones que cada actor institucional llevará a cabo. en un acta-acuerdo durante una reunión mantenida con la mamá de Manuel antes de su egreso. Las denominadas actas-acuerdo constituyen documentos que cristalizan la producción de consensos entre agentes y familias, y resultan centrales en el marco de abordajes caracterizados por la corresponsabilidad ${ }^{19}$ (Barna, 2015). En tal sentido, si bien no adquieren carácter coercitivo, señalan el curso del desarrollo de un caso al establecer tareas y responsables cuyo grado de cumplimiento establecen los parámetros sobre los cuales se definirá el éxito en la gestión de la situación. 
Ahora bien, ninguno de los acuerdos pudo sostenerse en el tiempo. Ni la mamá ni las tías de Manuel sostuvieron la asistencia al espacio de Terapia Multifamiliar luego de su egreso; el adolescente no asistía de manera regular al Centro de Día y cuando lo hacía provocaba "desmanes" y "situaciones violentas" como robos y amenazas con armas punzantes a compañeros y miembros del equipo de trabajo del lugar. En noviembre, esta institución informa al Servicio Local que, luego de evaluar "el extremo riesgo para sí como para el resto del personal y pares, desde el centro de día se radicó la denuncia correspondiente" (Fragmento de respuesta a oficio judicial del Juzgado de Garantías $\mathrm{N}^{\circ} 1$, noviembre de 2018, extraído del legajo del adolescente, p. 82). Adicionalmente, durante el transcurso de este mismo mes, desde el Juzgado de Familia se traslada al Servicio Local un petitorio elevado por la Junta Vecinal del barrio en el cual se encontraba viviendo Manuel:

La Junta Vecinal del barrio [...] desea canalizar mediante quien corresponda se evalúe INTERNACION del menor referido, en algún centro asistencial con el fin de evitar un mal mayor, y salvaguardar la integridad física de los vecinos del Barrio en mención y el menor en cuestión. (Fragmento de petitorio de Junta Vecinal, trasladada por medio de oficio judicial, diciembre de 2018, énfasis en el original, extraída del legajo del adolescente, p. 95.)

Ante lo informado por el centro de día y el petitorio trasladado desde el Juzgado, en el Servicio Local acuerdan con la mamá de Manuel que lo mejor sería que el adolescente ingresara a una "comunidad terapéutica" fuera de la localidad para evitar que el adolescente se fugue para volver al barrio, ya que consideraban previsible que esto sucediera.

Mientras transcurría la búsqueda de vacante en una comunidad terapéutica, durante los días finales del año, el Servicio Local es notificado nuevamente por el juzgado de un episodio de violencia con armas protagonizado por Manuel. Ante la imposibilidad de Laura y Felipe de acompañarlo al hospital - se había decidido su evaluación en el área de Salud Mental- debido a la resistencia del adolescente, el juzgado dispone su "internación involuntaria", esto es, haciendo uso de la fuerza policial. Al mismo tiempo, resuelve su alojamiento temporal en esta área del hospital como "espacio de término medio hasta tanto se resuelva la vacante en comunidad terapéutica" (Fragmento de oficio judicial del Juzgado de Familia $\mathrm{N}^{\circ} 1$, diciembre de 2018, extraído del legajo del adolescente, p. 99) a pesar de la insistencia de los médicos de que el Servicio Local dispusiera de otro lugar, como un hogar de guarda, debido a la ausencia de criterio médico para su estadía allí.

Durante el mes de enero de 2019, Manuel abandona el hospital en dos ocasiones, la primera de ellas pudo revertirse mediante intervención de su madre. La segunda ocasión implicó una denuncia por parte de ella misma, puesto que, acompañada por la policía a la casa de la abuela paterna del adolescente, donde creyó encontrarlo, no lo halló. De este modo, en la denuncia solicita la "intervención inmediata de la justicia" explicando que "su hijo debía internarse en los días siguientes en una comunidad terapéutica" (Fragmento de "denuncia policial", enero de 2019, extraída del legajo del adolescente, p. 121), ya que, efectivamente, había surgido una vacante. Finalmente, Manuel es localizado y, días más tarde, trasladado a una comunidad terapéutica en otra localidad.

Cuando se resuelve la adopción de la medida de seguridad que separa a Manuel de su familia, se efectúa por considerar a esta última responsable de las conductas y comportamientos "peligrosos" del adolescente. Al mismo tiempo, sus familiares más cercanos (madre, tías y abuela) adquieren cierta disposición ante lo sucedido, y se posicionan. Demuestran predisposición a colaborar y comprometerse con respecto a lo que los diferentes agentes institucionales les solicitan, porque entienden esto como condición 
20. Me inspiro para el desarrollo de este argumento en lo que Lugones (2012) denomina como formas de aconsejamiento. Pero más precisamente me refiero aquí a los consejos que no solo procuran que las familias hagan lo que los profesionales les aconsejan, sino sobre todo que piensen que hacer eso es realmente lo mejor o lo más conveniente.

21. Agustín Barna (2014b) sostiene que las relaciones entre los dispositivos administrativos de gestión de la infancia y las unidades domésticas, en el marco de los actuales formatos de intervención social sobre la niñez considerada "en riesgo", tienen más de negociación y complementariedad asimétrica que de un control vertical ejercido por los agentes estatales sobre las poblaciones. Se trata de interacciones, señala el autor, que hablan de la configuración de relaciones de singular complementariedad a las que denomina "alianza asimétrica" para ilustrar su doble dimensión, "en cuanto negociación activa de los diversos actores involucrados, pero inserta en un determinado entramado de relaciones de poder" para recuperar a Manuel. Sin embargo, para los profesionales responsables del caso, el regreso a su ámbito familiar parecía haber promovido un retorno a las conductas "riesgosas" que habían provocado su institucionalización. En tal sentido, el problema persistía en el ámbito familiar, e incluso su madre bregaba por la no permanencia de su hijo en este ámbito.

El escenario configurado se presentaba por demás dilemático: las propias instituciones que debieran aunar esfuerzos para proteger a Manuel parecían desligarse de esta tarea, disputaban los alcances de sus intervenciones e incluso ellas mismas llegaron a denunciarlo. Pero lo que parecía muy claro, tanto para el Servicio Local como para la mamá de Manuel, era que el adolescente no podía continuar viviendo con su familia, ya fuera en el hogar materno o en casa de su abuela. Si bien en un principio la familia de Manuel apeló al regreso del adolescente a su hogar, asumiendo la responsabilidad por la situación y ofreciendo incluso el ámbito alternativo de la vivienda de su abuela, finalmente esta posición se revierte ante la imposibilidad manifestada por su mamá de "hacerse cargo" de su hijo. Entonces, aquí resulta necesario advertir que el carácter dilemático que adquiere la situación se configuraba no solo para quienes gestionaban el caso, sino particularmente para la mamá de Manuel, sobre quien recaía el peso de la responsabilidad atribuida y moldeada institucionalmente. En tal sentido, más allá de las dificultades surgidas para apuntalar los comportamientos del adolescente, entiendo que el cambio de parecer de su mamá no surge como consecuencia única de las actitudes de su hijo. El trabajo de Laura y Felipe también, y sobre todo, había producido efectos sobre lo que la familia consideraba "lo mejor" para Manuel. Si en un principio el empeño estaba puesto en "mejorar" para lograr su regreso al ámbito familiar, ahora su madre estimaba que lo conveniente era procurar su "internación".

Resulta oportuno advertir aquí acerca de la ausencia en los documentos que constituyen mi material de análisis sobre el caso, tanto de la voz de la abuela en esta instancia del proceso como de lo que puede denominarse un "trabajo de convencimiento" sobre las familias. ${ }^{20}$ En términos de Vianna (2002), estos documentos reflejan las "verdades parciales" (p. 275) que los procesos encierran, y dan cuenta del carácter de objetos socialmente construidos que portan, pero también de objetos socialmente constructores, creadores de realidades. Entonces, la ausencia de la voz de la abuela en ellos da cuenta de lo que la autora denomina una "economía de la mediación" en las actuaciones de los profesionales, y de una "economía lingüística" que permite "dejar registrada la voz de las personas o suprimirlas, construyendo a través de las supresiones un lugar para posibles negociaciones" (p. 295). Así, este cambio en el parecer de la mamá de Manuel aparece registrado bajo la forma de acuerdo consensuado y como decisión consecuente de las actitudes y comportamientos del adolescente. A su vez, el andamiaje de interacciones efectuadas que llevan a la decisión final de la institucionalización del joven devela el carácter asimétrico de la alianza agentes-familias (Barna, 2014b), ${ }^{21}$ en tanto se trata de una decisión que se construye moldeada por el trabajo estatal de los profesionales. Un trabajo que, caracterizado por el empleo de herramientas asociadas al diálogo y el consenso, pareciera disipar u ocultar el ejercicio de poder estatal presente en estas actuaciones. Al no poder asumir el rol que desde las instituciones se le atribuye con respecto al bienestar de su hijo, la mamá del adolescente encuentra que la institucionalización parecía ser la única vía disponible que ofrecía el Estado como modo de resguardo para su hijo. En tal sentido, entonces, asume una imposibilidad, adjudicada desde el mismo Estado, de proteger a su hijo y delega en él la tarea.

En una entrevista realizada días previos al ingreso de Manuel en la comunidad terapéutica, su madre expresa estar totalmente de acuerdo con la institucionalización de su hijo porque "se le va de las manos", "no puede controlarlo" y "no quiere que vuelva a su hogar porque representa un peligro para él". (Diario de campo, entrevista a la madre de Manuel en sede del Servicio Local, enero de 2019). Sin embargo, tal como refieren 
Laura y Felipe, "ella no se desentiende de su rol como madre, no pretende desligarse de su hijo, lo acompaña todo lo que puede y lo que su relación con él se lo permite". (Diario de campo, testimonio de agentes del Servicio Local, diciembre de 2018). En este sentido, entiendo la posición última de la mamá de Manuel como un modo de "expresión pública" (Stagno, 2019) de las dificultades que experimentaba para ocupar el rol social y moral que se le atribuía. Sin embargo, resulta importante dar cuenta de que en el relato de los profesionales se evidencia un intento de calificar moralmente la actitud de la mamá de Manuel debido a que no se trataba de "abandono" o "irresponsabilidad", sino que su actitud se encuadraba dentro de un orden institucionalmente válido e incluso necesario para llevar adelante las actuaciones deseadas.

De modo que, así configurado el escenario, la institucionalización, más que impuesta, tomaba la forma de estrategia negociada y consensuada. E incluso es la propia madre del adolescente quien esgrime esta posibilidad como herramienta para vehiculizar lo que interpreto como estrategia familiar de distribución de responsabilidades entre familia y Estado. Con esto busco mostrar que los sentidos dados a la institucionalización, como medida de última opción en el ámbito de la administración de la infancia, pueden ser muchas veces puestos en tensión por familias que, en la imposibilidad de asumir los roles socialmente asignados, la convierten en recurso para su propia administración familiar. Y sin embargo, aunque pareciera ser estrategia consensuada e incluso solicitada por la mamá de Manuel, está claro que en el afán regulatorio y normalizador de las relaciones familiares es el propio Estado el que demarca la posibilidad de estos usos de la institucionalización. Aun así, en este tipo de negociación que signa las modalidades actuales de gestión de la niñez puede advertirse la vehiculización del ejercicio de poder estatal que conducía e incluso producía las respuestas que la mamá de Manuel ofrecía, y que reproduce antes que salva las desigualdades.

\section{Consideraciones finales}

En este trabajo se han abordado distintos escenarios dilemáticos que comúnmente se configuran a raíz de las intervenciones sobre la infancia y sus familias enmarcadas en las actuales modalidades de protección de derechos de niñas, niños y adolescentes. Focalizando el análisis en la reconstrucción de un caso abordado en un Servicio Local de una localidad de rango medio de la provincia de Buenos Aires, intenté mostrar los modos en que estas intervenciones traducen situacionalmente el lenguaje de derechos a las realidades particulares en las cuales se inscriben. De este modo di cuenta de la resignificación que este lenguaje adquiere en las intervenciones concretas, a partir de las tensiones que nociones como consenso, voluntariedad y responsabilidad familiar producen en su inscripción en las prácticas reales que encarnan los derechos. Nociones que conviven y se imbrican con singulares reconversiones del poder tutelar sobre la infancia y sus familias. Así, por ejemplo, encauzar los comportamientos desviados de Manuel implicó, sobre todo, componer un orden familiar que aceptara, primero, modificar sus comportamientos y modos de relacionamiento, y luego, al no lograr esto, aceptar e incluso solicitar el control directo sobre las conductas del adolescente.

Si bien la institucionalización no aparece como objetivo explícito ni primario, el esbozo de su posibilidad se presentaba por medio de un abordaje de la situación caracterizada por el empleo de técnicas asociadas a la persuasión, el diálogo y la elaboración de acuerdos. Puede interpretarse esto como una especie de magia social (Mauss, 1979; Bourdieu, 1985) cuya eficacia radica en una apariencia (Bourdieu, 1985, p. 71), esto es, en la ilusión de hacer creer como voluntarias, decisiones, acciones y deseos moldeados por el principio de un poder contenido en las propias condiciones de institucionalidad de la relación Estado/familias. 
Las situaciones analizadas nos muestran que, desde el momento en que es definido el "problema", la selección de elementos a ponderar y otros a ocultar u obviar muestra el carácter construido tanto de dicho problema como del objeto de intervención. Y ello porque la persistencia de evaluaciones morales sobre los roles familiares en el campo institucional de la protección de la infancia, permanece por encima de los condicionamientos de carácter estructural que permean las relaciones sociales en contextos de desigualdad. Las diversas evaluaciones y clasificaciones (Barna, 2014a) que operaron sobre el proceso que derivó en la institucionalización de Manuel responden a una moralización de las relaciones y comportamientos familiares que, a su vez, son moldeadas por categorías propias del campo burocrático que caracterizan y definen las problemáticas. Así, "conflictiva vincular", "modos de vinculación violenta" o "conductas transgresoras" constituyen categorías que enmarcan y encauzan problemas, los producen y moldean mediante fórmulas burocráticas habituales (Villalta, 2010). Sin embargo, las situaciones analizadas fueron traducidas a un lenguaje que no abarca la compleja realidad que envuelve los hechos. Esto es, el recorte individualista de los problemas y conflictos sobre las personas (Schuch, 2008; Magistris, 2011) deja por fuera todo un contexto de vulnerabilidades que hacen a estos problemas. Entonces, entiendo que lo que parecieran ser versiones incompletas de lo que sucede en la vida social, acotadas al ámbito de las regulaciones familiares, revelan en realidad un modo de clasificación de personas y problemáticas pasibles de ser abordadas por determinadas agencias estatales. Como señala Villalta (2010), se trata de un campo institucional que crea su propio objeto de intervención.

Al recortar la problemática exclusivamente sobre las personas involucradas, al mismo tiempo se les asigna la responsabilidad por revertirla. Vimos que las intervenciones se abocaban exclusivamente a lograr que tanto el adolescente como su familia cambiaran hábitos, conductas y comportamientos considerados "peligrosos" por medio de instancias de autotransformación, como son los diversos espacios terapéuticos a los que debían asistir. Sin embargo, la asignación de responsabilidades (Gluckman, 1972) sobre la familia de Manuel, fundamentalmente sobre su madre, no solo en cuanto a la modificación de sus conductas, sino también por la institucionalización del adolescente derivó en una modificación del modo en que la familia se había posicionado frente a la situación. Considerando a la familia como una organización activa frente a las tramas de intervenciones que sobre ella se despliegan (Santillán, 2013), el margen de maniobra y negociación del que disponen para ofrecer sus respuestas a estas acciones se reconfigura en los escenarios analizados. En el esfuerzo por componer soluciones a los problemas, los profesionales construyen para las familias un orden de experiencia informada por moralidades capilarizadas en el saber técnico y las actuaciones de los agentes. En tal sentido, la opción de la institucionalización finalmente es representada como recurso o estrategia de repartición de responsabilidades entre la familia y el organismo administrativo.

Así, los sentidos que la institucionalización adquiere en la situación particular analizada dan cuenta de la no unidireccionalidad de las intervenciones estatales y de las formas en que las propias familias hacen uso del lenguaje de derechos en su traducción situacional. Procesos como el analizado son tensionados y disputados por medio de del despliegue que las familias pueden realizar de sus propias acciones, pero insertas en un determinado entramado de relaciones de poder. De modo que los efectos que estos dilemas producen tienden a una resignificación del lenguaje de los derechos alimentado por las expectativas, respuestas y acciones que las familias pueden proyectar en su interacción con el Estado. 


\section{Financiamiento}

Este artículo presenta resultados de una investigación desarrollada en el marco de una beca del Consejo Interuniversitario Nacional EVC-CIN por el período 2018-2019, con asiento en el Grupo de Estudios Socioculturales del Conflicto (FACSO-UNICEN), dirigido por el Dr. Juan Pablo Matta.

\section{Agradecimientos}

Agradezco especialmente al Dr. Agustín Barna por la lectura atenta de las versiones preliminares de este artículo y por su comprometido acompañamiento como director de la investigación que enmarca este trabajo.

\section{Biografía}

Licenciada en Antropología Social (FACSO-UNICEN). Doctoranda en Antropología (Facultad de Filosofía y Letras-Universidad de Buenos Aires). Becaria doctoral (CONICET). 


\section{Q Referencias bibliográficas}

" Barna, A. (2014a). No hay como la palabra escrita para defender lo que uno hace: Usos de la producción documental en la gestión cotidiana de la niñez en el municipio de La Matanza. Intersecciones en Antropología, 15, 153-165.

" Barna, A. (2014b). Relaciones entre dispositivos administrativos y arreglos familiares en la gestión de la infancia "con derechos vulnerados". Una aproximación etnográfica. Revista de Estudios Sociales, 50, 57-70. doi: http://dx.doi.org/10.7440/res50.2014.08

" Barna, A. (2014c). Clasificaciones y estimaciones en la gestión de la infancia "con derechos vulnerados". Prácticas cotidianas de intervención en un dispositivo estatal del conurbano bonaerense. Revista Antropolítica, 36, 113-148.

" Barna, A. (2015). Desentrañar sucesos, evaluar sujetos y producir verdades para "restituir derechos de niños". Runa, 36(1), 73-89.

» Bourdieu, P. (1985). ¿Qué significa hablar? Madrid: Akal.

"Ciordia, C. y Villalta, C. (2012). Procesos judiciales y administrativos de adopción de niños: confrontación de sentidos en la configuración de un "medio familiar adecuado". Etnográfica, 16(3), 435-460.

»Douglas, M. (1996). La aceptabiliad del riesgo según las ciencias sociales. Barcelona: Paidós.

»Fonseca, C. y Cardarello, A. (2005). Derechos de los más y menos humanos. En S. Tiscornia, Sofía y M. Pita (Eds.). Derechos humanos, tribunales y policías en Argentina y Brasil. Estudios de antropología jurídica (pp. 9-41). Buenos Aires: Antropofagia.

» Gluckman, M. (1972). The allocation of responsibility. Manchester: Manchester University Press.

"Grinberg, J. (2008). Transformaciones en el tratamiento de la niñez en riesgo. Reflexiones sobre un dispositivo de protección a la infancia en la Ciudad de Buenos Aires. Cuadernos de Antropología Social, 27, 155-174.

" Grinberg, J. (2013). La recepción de "los derechos del niño" en Argentina: trayectorias de activistas y conformación de una nueva causa en torno a la infancia. Virajes, 15(1), 299-325.

" Grinberg, J. (2018). "Con tantos casos resulta difícil, además los padres reaccionaron bien y seguir interviniendo sería demasiado intrusivo": reflexiones sobre las formas de regulación de las relaciones familiares en tiempos de la protección integral de derechos de la infancia y la adolescencia. En stas. Jornadas de Estudios sobre la Infancia, Buenos Aires. Recuperado de https://www.aacademica.org//jornadasinfancia/22

» Lugones, M. G. (2012). Obrando en autos, obrando en vidas: formas y fórmulas de protección judicial en los tribunales prevencionales de menores de Córdoba, Argentina, a comienzos del siglo XXI. Río de Janeiro: E-Papers.

» Magistris, G. (2011). Políticas de protección de derechos de niñas, niños y adolescentes en la provincia de Buenos Aires: entre disputas, conflictos y apropiaciones. $X$ Congreso Nacional de Ciencia Política. Democracia, integración y crisis en el nuevo orden global. Sociedad Argentina de Análisis Político - Universidad Católica de Córdoba, Córdoba, Argentina. Recuperado de https://www.aacademica.org/gabrielamagistris/2

" Mauss, M. (1979). Esbozo de una teoría general de la magia. En Sociología y Antropología. (pp. 56-148). Madrid: Tecnos. 
»Merry, S. E. (2010). Derechos humanos y violencia de género: el derecho internacional en el mundo de la justicia local. Bogotá: Universidad de los Andes.

" Muzzopappa, E. y Villalta, C. (2011). Los documentos como campo. Reflexiones teórico metodológicas sobre un enfoque etnográfico de archivos y documentos estatales". Revista Colombiana de Antropología, 47(1), 13-42.

»Pacheco de Oliveira, J. (1988). "O Nosso Governo”. Os ticunas e o Regime Tutelar. Río de Janeiro: Marco Zero.

"Santillán, M. L. (2013). Entre la ayuda y el “desligamiento". Prácticas y regulaciones cotidianas sobre las familias y el cuidado infantil en barrios populares del Gran Buenos Aires - aportes desde la etnografía. Civitas, 13(2), 326-345.

"Schuch, P. (2008). Tecnologias da não violência e modernização da justiça no Brasil. Civitas, 8(3), 498-520.

"Souza Lima, A. C. (2009). Poder tutelar y formación del Estado en Brasil: notas a partir de la creación del Servicio de Protección a los Indios y Localización de Trabajadores Nacionales. Desacatos, 33, 53-66.

"Stagno, L. (2019). Una cultura juvenil callejera: Sociabilidades y vida cotidiana de varones jóvenes en la ciudad de La Plata (1937-1942). En M. P Bontempo y A. Bisso (Eds.), Infancias y juventudes en el siglo XX: política, instituciones estatales y sociabilidades (pp. 189-213). Ciudad Autónoma de Buenos Aires: Teseo Press.

»Vianna, A. (2002). Quem debe guardar as crianças? Dimensões tutelares de gestãoda infância. En A. C. Souza Lima, Gestar e gerir. Estudos para uma antrpologias administração pública no Brasil (pp. 271-311). Río de Janeiro: Relume-Dumará.

"Vianna, A. (2010). Derechos, moralidades y desigualdades. Consideraciones acerca de procesos de guarda de niños. En C. Villalta (Comp.), Infancia, justicia y derechos humanos (pp. 21-72). Buenos Aires: Universidad Nacional de Quilmes.

"Villalta, C. (2010). La administración de la infancia en debate. Entre tensiones y reconfiguraciones institucionales. Estudios de Antropología Social, 1(2), 81-99.

》Villalta, C. (2012). Entregas y secuestros: el rol del Estado en la apropiación de niños. Ciudad Autónoma de Buenos Aires: Del Puerto.

»Villalta, C. (2013). Un campo de investigación: las técnicas de gestión y los dispositivos jurídico-burocráticos destinados a la infancia pobre en la Argentina. Civitas, 13(2), 235258.

"Villalta, C. y Llobet, V. (2015). Resignificando la protección. Nuevas normativas y circuitos en el campo de las políticas y los dispositivos jurídico-burocráticos destinados a la infancia en la Argentina. Revista Latinoamericana de Ciencias Sociales, Niñez y Juventud, 13(1), 167-180.

\section{Otras fuentes consultadas}

" Informe de Dirección de Psicología Comunitaria y Pedagogía Social y Dirección de Educación Primaria, municipio de Olavarría. Noviembre 2015. Extraído de: Legajo del caso, correspondiente al Servicio Local de Promoción y Protección de Derechos del Niño de Olavarría, Buenos Aires, p. 4-5.

» Informe de situación. Marzo 2018. Extraído de: Legajo del caso, correspondiente al Servicio Local de Promoción y Protección de Derechos del Niño de Olavarría, Buenos Aires, p. 20-21. 
» Informe psicológico del Servicio de Pediatría, área Salud mental, hospital municipal. Marzo 2018. Extraído de: Legajo del caso, correspondiente al Servicio Local de Promoción y Protección de Derechos del Niño de Olavarría, Buenos Aires, p.3o.

» Oficio judicial: acta de audiencia en Juzgado de Garantías del Joven № 1 de Azul. Mayo 2018. Extraído de: Legajo del caso, correspondiente al Servicio Local de Promoción y Protección de Derechos del Niño de Olavarría, Buenos Aires, p.59-6o.

» Informe de situación. Junio 2018. Extraído de: Legajo del caso, correspondiente al Servicio Local de Promoción y Protección de Derechos del Niño de Olavarría, Buenos Aires, p.63-64.

» Acta de comunicación telefónica. Junio 2018. Extraído de: Legajo del caso, correspondiente al Servicio Local de Promoción y Protección de Derechos del Niño de Olavarría, Buenos Aires, p.58.

"Informe de Terapia Multifamiliar. Junio 2018. Legajo del caso, correspondiente al Servicio Local de Promoción y Protección de Derechos del Niño de Olavarría, Buenos Aires, p.66-71.

»Respuesta a oficio judicial del Juzgado de Garantías $\mathrm{N}^{\circ}{ }_{1}$. Noviembre 2018. Extraído de: Legajo del caso, correspondiente al Servicio Local de Promoción y Protección de Derechos del Niño de Olavarría, Buenos Aires, p.82.

» Petitorio de Junta Vecinal Juan Martín de Pueyrredón. Diciembre 2018. Extraída de: Legajo del caso, correspondiente al Servicio Local de Promoción y Protección de Derechos del Niño de Olavarría, Buenos Aires, p.95.

» Oficio judicial del Juzgado de Familia $N^{\circ}$ 1. Diciembre 2018. Extraído de: Legajo del caso, correspondiente al Servicio Local de Promoción y Protección de Derechos del Niño de Olavarría, Buenos Aires, p. 99.

»Denuncia policial. Enero 2019. Extraída de: Legajo del caso, correspondiente al Servicio Local de Promoción y Protección de Derechos del Niño de Olavarría, Buenos Aires, p. 121. 